\title{
Depth of origin of sputtered atoms: Exploring the dependence on relevant target properties to identify the correlation with low-energy ranges
}

\author{
Klaus Wittmaack ${ }^{\mathrm{a}^{*}}$, Andreas Mutzke ${ }^{\mathrm{b}}$ \\ ${ }^{a}$ Helmholtz Zentrum München, Institut für Strahlenschutz, D-85758 Neuherberg, Germany \\ ${ }^{b}$ Max-Planck-Institut für Plasmaphysik, EURATOM Association, Teilinstitut Greifswald, D-17491 \\ Greifswald, Germany
}

The correlation of the depth of origin (DoO) of sputtered atoms with relevant properties of the bombarded target has not yet been clarified in a satisfactory manner. We have carried out SDTrimSP (Monte Carlo) simulations to study the dependence of depth differential sputtering yields on the following parameters: the position of the primary knock-on atom, the surface binding energy, $E_{\mathrm{s}}$, the target density, $N$, and the target atomic number, $Z_{2}$. All calculations were performed with normally incident projectiles, either Xe or targetatom-like, with energies between $40 \mathrm{eV}$ and $10 \mathrm{keV}$. The mean DoO, $\langle x\rangle$, was found to depend on $E_{\mathrm{s}}$ as $\langle x\rangle \propto E_{\mathrm{s}}^{\mathrm{p}}$, with a power $p$ between $0.10(0.1 \mathrm{keV})$ and $0.26(10 \mathrm{keV} \mathrm{Xe})$. In prior work, lack of knowledge of this weak $E_{\mathrm{s}}$-dependence did not allow the relation between $\langle x\rangle$ and $N$ to be uncovered properly. To proceed further, differential yields were calculated for targets ranging from carbon to uranium with deliberately varied $N$, but keeping $E_{\mathrm{s}}$ fixed $(4.72 \mathrm{eV})$. The product $\langle x\rangle N$ turned out to depend very little on $N$. Hence, it is appropriate to specify the origin of sputtered atoms in terms of the equivalent areal density rather than in units depth. For medium-mass or heavy target atoms, i.e., for $Z_{2}>20,\langle x\rangle N$ was largely independent of $Z_{2}$. For $Z_{2}<20,\langle x\rangle N$ increased with decreasing $Z_{2}$. Projected ranges $x_{\mathrm{p}}$ calculated for targetatom-like projectiles at energies $\leq 40 \mathrm{eV}$ exhibited almost the same $Z_{2}$-dependence as $\langle x\rangle$. This finding suggests that $\langle x\rangle$ is determined by the range of the recoils mobilized in the collision cascade. The results of this study are compared with previously published data, with sometimes distinctly different definitions of $\langle x\rangle$. The idea is recapitulated that the most appropriate way of quantifying the $\mathrm{DoO}$ is to quote the fraction of atoms ejected from the top (or outermost) layer of atoms. The fractions determined by SDTrimSP range from about $98 \%$ to $75 \%$, for impact energies between $40 \mathrm{eV}$ and $10 \mathrm{keV}$ and $Z_{2}>20$.

Keywords: Sputtering, depth of origin, surface binding energy, density effect, projected range

\footnotetext{
${ }^{*}$ Corresponding author. Tel. : +49 893187 2439; fax: +49 8931872942

E-mail address: wittmaack@helmholtz-muenchen.de
} 


\section{Introduction}

The phenomenon of sputtering initiated by bombardment of a solid or liquid sample with energetic ions is often described merely in terms of the total yield $Y$. In surface analytical applications of sputtering as well as in theoretical studies, however, one likes to know where the atoms had been residing before they were ejected. Experimentally, the desired information is not easy to determine. One approach is to deposit small quantities of an element of choice (from sub-monolayer coverage up to about two monolayers) on a preferably crystalline substrate of a different material, and to monitor the signals due to sputter ejection of atoms or ions from the deposit and the substrate [1,2]. For minimum distortion of the sample, the applied primary ion fluence should be small $\left(<10^{12}\right.$ ions $\left./ \mathrm{cm}^{2}\right)$. A distinctly different approach is to use a liquid eutectic alloy as a sample, with two appropriately chosen constituents, one being present at the surface as a highly enriched monolayer [3]. The studies showed that the majority of the sputtered atoms originated in the topmost (outermost) atomic layer, more so the lower the primary ion energy. Supporting evidence was obtained from sputter depth profiles of a layer of ${ }^{30} \mathrm{Si}$ on ${ }^{28} \mathrm{Si}$, measured by secondary ion mass spectrometry (SIMS) and mass resolved ion scattering spectrometry (MARISS), the latter technique serving to monitor the surface composition quasi-simultaneously [4].

In theoretical studies, the distribution of the depth of origin of sputtered atoms is described by the depth differential sputtering yield, $\psi(x)$, defined as

$\psi(x)=d Y / d x$,

with $x$ being the depth normal to surface of the sample. Note that a definition as in Eq. (1) disregards the atomistic nature of the sample. At any surface position $(y, z)$, atoms can only originate either from the outermost or from some deeper layer, not from a deliberately chosen depth $x$. Hence, Eq. (1) constitutes a simplification that needs to be kept in mind when discussing results obtained with models involving continuously variable near-surface positions of atoms in a randomized target, like the Monte-Carlo code TRIM.SP [5]. In the programs MARLOWE [6] and OKSANA [7], an amorphous sample is simulated by rotating, after each impact event, the crystalline blocks of target atoms.

Rather than discussing $\psi(x)$, calculated data are commonly expressed in terms of the mean depth of origin (DoO), $\langle x\rangle$, i.e., 
$\langle x\rangle=Y^{-1} \int_{0}^{\infty} x \psi(x) \mathrm{d} x$

with

$Y=\int_{0}^{\infty} \psi(x) \mathrm{d} x$

The mean DoO is an important quantity in the analytical transport theory of sputtering [8]. The predicted number is sensitive to the absolute value of the stopping cross section, calculated for Born-Mayer interaction. The required fitting procedure was refined in later work [9]. One may circumvent the problem by casting the predicted yield [8] into a form such that $\langle x\rangle$ becomes an input parameter [10],

$Y(E)=\frac{\langle x\rangle N}{\pi^{2} E_{\mathrm{s}}} \alpha S_{\mathrm{n}}(E)$

where $N$ denotes the number density of target atoms. $E_{\mathrm{s}}$ is the surface binding energy and $S_{\mathrm{n}}$ the nuclear stopping cross section for slowing-down a projectile of energy $E$, atomic number $Z_{1}$, and mass $M_{1}$ in a target composed of atoms $Z_{2}, M_{2}$. The energy actually deposited near the surface depends on the structural details of the collision cascades evolving as a result of the primary ion impacts. The net efficiency of this transfer of kinetic energy is condensed in the dimensionless factor $\alpha$ which is usually assumed to depend only on the mass ratio $M_{2} / M_{1}[8,10,11,12]$. Eq. (4) does not include threshold effects observed at impact energies below about ten times the threshold energy for sputtering, which ranges from ca. 10 to $100 \mathrm{eV}[10,11,13]$.

In Eq. (4), the product $\langle x\rangle N$, i.e. the areal density of target atoms contained within $\langle x\rangle$, constitutes the 'source' of sputtered atoms. One of us (KW) has shown that Eq. (4) may in fact be used to reproduce measured sputtering yields with an accuracy of typically $\pm 20 \%$ or better [10]. To achieve this kind of agreement, $\langle x\rangle N$ had to be interpreted as the sum of the (apparent) mean DoO, $x_{0} N$, reported in [4], and the areal density of the outermost layer to which a thickness equal to the mean atomic spacing $\lambda_{0}=N^{-1 / 3}$ was assigned,

$\langle x\rangle N=x_{0} N+N^{2 / 3}=\left(x_{0}+\lambda_{0}\right) N$.

Several unsolved problem need to be addressed. (i) In Eq. (4), $\langle x\rangle$ is a constant [14], i.e., independent of the primary ion energy. According to simulations by MARLOWE [6] as well as by TRIM.SP and OKSANA [5], however, $\langle x\rangle$ increases with increasing impact energy as $E^{q}$. Though the power $q$ is small ( 
0.17 according to the data in [5]), a change of $E$ by a factor of 100 results in an increase of $\langle x\rangle$ by a factor of about two. An energy dependence of $\langle x\rangle$ is also evident in data obtained by MARLOWE [6] as well as by molecular-dynamics (MD) simulations using the SPUT2 code [15]. (ii) There are significant differences in defining the origin of the depth scale [15]. To ease comparison of $\langle x\rangle$-data obtained by TRIM.SP and SPUT2, it has been suggested [15] to 'correct' the MD results by adding $\lambda_{0} / 2$ (in MD simulations, $\langle x\rangle=0$ is assigned to atoms ejected from the outermost layer). (iii) Based upon the idea that the position at which target atoms are located prior to being ejected in a sputtering event should be quoted in units of depth, much work has been devoted to clarifying the correlation between $\langle x\rangle$ and the target density $N$. Kelly and Oliva [16] analyzed experimental data to conclude that $\langle x\rangle \propto \lambda_{0}=N^{-1 / 3}$. TRIM.SP and OKSANA simulations by Shulga and Eckstein [5] suggested a relation of the form $\langle x\rangle \propto N^{-k}$ with $k \cong 0.86$. A closer inspection of Fig. 13 in [5], however, shows that this is gross approximation which ignores at least two important features of the data. First, the $\langle x\rangle$-values for the light-element targets $\mathrm{Li}, \mathrm{Be}, \mathrm{B}$ and $\mathrm{C}$ are significantly higher than for medium-mass and heavy elements. Second, ignoring the light elements in a data re-evaluation, the power $k$ turns out not to be constant but to increase with decreasing impact energy $E$, from about 0.85 for $10 \mathrm{keV} \mathrm{Ar}$ to about 1.0 for $100 \mathrm{eV} \mathrm{Ar}$ (normal incidence, TRIM.SP data only [5]). SPUT2 simulations by Shapiro et al. [15] for $5 \mathrm{keV}$ Ar yielded $k=0.80$ and 0.57 for (100) and (111) targets, respectively.

Remarkably, the effect of the surface binding energy $E_{\mathrm{s}}$ on $\langle x\rangle$ was not explored in the previous work $[5,15]$. Given the fact that the sputtering yield depends inversely on $E_{\mathrm{s}}$, see Eq. (4), it would be quite surprising if $\langle x\rangle$ were completely independent of $E_{\mathrm{s}}$. Clarifying the issue was the first aim of this simulation study. In that context we also expected to arrive at an understanding of the somewhat strange and controversial $N$-dependence of $\langle x\rangle[5,15,16]$ summarized above. Last but not least, it appeared promising to take a second look at the variation of $\langle x\rangle$ with target atomic number, the ultimate goal being a reasonably complete description of the functional dependence $\langle x\rangle=f\left(E, E_{\mathrm{s}}, N, Z_{2}\right)$.

\section{Some relevant features of SDTrimSP}

Depth differential sputtering yields and ranges were calculated using the Monte-Carlo simulation code SDTrimSP [17]. The program combines the former static version TRIM.SP [18] and the dynamic version 
TRIDYN [19] without changing the basic physics. In the notation SDTrimSP the first two letters stand for static (S) and dynamic (D). The user of the program has to select the version of interest, S or D. Irrespective of this choice, the program assumes an amorphous (randomized) target at zero temperature, laterally of infinite size, but of limited (deliberately chosen) thickness. Elastic (nuclear) collisions between atoms are treated in the binary collision approximation, i.e., changes in flight direction are represented by the asymptotes of the real trajectories. The scattering angle of the projectile or a moving atom and the recoil angle of the struck atom as well as the corresponding energy loss and gain, respectively, are determined by the chosen interaction potential. The (inelastic) energy loss to target electrons is also calculated. The program follows the trajectories of all atoms set in motion until their energy has fallen below some preset value, referred to as the cut-off energy $E_{\text {cut }}$, or until the respective atoms may have left the target (backscattered or transmitted projectiles, sputtered target atoms).

An important feature of the new program is the modular structure which provides more flexible output and higher portability. It runs on all sequential and parallel platforms with a Fortran 90 compiler. Most of the data needed for the calculations are taken from a data base in the form of tables, containing atomic numbers and masses of elements (isotopes), liquid and solid densities, inelastic energy losses etc. More details are described in [17].

To ease understanding of the results presented below, it appears appropriate to briefly discuss some specific features of the code. The surface of the sample is represented by the $y, z$-plane located at depth $x=0$. The composition and the density of the target are input parameters. The centre of the first target atom, the primary knock-on atom, represented by atom $\mathrm{P}$ in Fig. 1, may be placed at $x=0$, as shown, or at a randomly selected position in the range $0 \leq x \leq . \lambda_{0}$. Other target atoms do not exist initially. They are created, step by step, only after the first atom has been set in motion. The next collision partner is assumed to be located on a sphere, at a distance equivalent to the mean free path $\lambda_{0}$. The shortened version of $\lambda_{0}$ suggested by Shulga and Eckstein was not adopted [5] because the resulting effect is contained in the systematic changes of the target density explored in the present study. The actual location of the next collision partner in terms of azimuthal angle and impact parameter are determined by a random number generator [17]. This procedure is carried on for the projectile on its way into the 'target' as well as for all recoil atoms. 
Consider now the series of recoil events sketched in Fig. 1. For book keeping in static SDTrimSP, the target is divided into layers of thickness $\Delta x$, either some fraction of $\lambda_{0}$ (example in Fig. 1: $\lambda_{0} / 8$ ) or a fixed number in standard units of depth ( $\AA$ or nm). The grey arrow near the bottom is meant to represent energy and momentum that has been transported by the collision cascade in the direction of the surface. As a result, atom $\mathrm{A}$ was set in motion. The random number generator created atom $\mathrm{B}$ at distance $\lambda_{0}$, located on the dashed circle around the centre of A (all collision events are projected into the plane of drawing). The subsequent collisions B-C, C-D, and D-F terminated in the sputter ejection of atom F located in the depth interval $0 \leq x \leq \lambda_{0} / 8$, i.e., very close to the nominal surface. Such a hypothetical position is only possible in the simulation and a consequence of definition of the surface rather than by taking into account the true local arrangement of atoms at the point of ejection. Owing to their random creation, target atoms can exist at any position $x \geq 0$. Hence, summing up over a sufficiently large number of impact events, all depth intervals $\Delta x$ below the surface will be more or less uniformly filled with target atoms (up to the maximum range of the projectile). In Fig. 1, some additional target atoms are schematically represented by circles with thin lines.

Recoil atoms will frequently propagate off-normal, i.e., including small angles with respect to the surface. An example is atom D in Fig. 1. If we interpret $\lambda_{0}$ as the thickness of one monolayer, off-normal motion has the important consequence that atoms can experience sizable energy loss as they travel through a region that is nominally equivalent the topmost layer of the sample. This special feature of TRIM simulations may be expected to show up somehow in the simulated data.

\section{Numerical details}

In this study, SDTrimSP was operated exclusively in the static mode, meaning that each impact event was started with a 'fresh' target. The $\mathrm{KrC}$ potential [20] and a planar surface potential were used throughout. Xe atoms were chosen as standard projectiles; additional calculations were performed with $M_{1}=M_{2}$, all at normal incidence $\left(0^{\circ}\right)$. The following targets were selected: $\mathrm{C}, \mathrm{Si}, \mathrm{Ti}, \mathrm{Ge}, \mathrm{Ag}, \mathrm{Cs}, \mathrm{W}$, and $\mathrm{U}$. The target and projectile properties ( $N, E_{\mathrm{s}}$ and mean masses of the isotopes) were either taken from Table 6.1 in Ecksteins's monograph [21] or were chosen deliberately. Most of the data were stored with $\Delta x=0.05 \mathrm{~nm}$, a few with $0.02 \mathrm{~nm}$. Depending on the required statistical accuracy, the minimum number of projectiles was $1 \times 10^{6}$. 
Significant attention was paid to the numerical accuracy of the calculations. Different integration methods for evaluating the scattering integral were tested, Magic [22], Gauß-Mehler [23] and GaußLegendre [24]. Magic is known to have the advantage of being faster than the other integration techniques [20]. One set of results for the differential sputtering yield of Si bombarded with $10 \mathrm{keV} \mathrm{Xe}$ is presented in Fig. 2 for $E_{\mathrm{s}}=4.72 \mathrm{eV}$ (standard value for $\mathrm{Si}$ ). In another run $E_{\mathrm{s}}$ was deliberately reduced to $0.2 \mathrm{eV}$, for test purposes. Calculations with Gauß-Mehler (GM) and Gauß-Legendre (GL) were done using 8 or 16 supporting points, labelled GM8, GM16 and GL8. For ease of comparison the data in Fig. 2 are also shown as ratios (asterisks and crosses). Whereas the differential yields for GM8 and GM16 agree with GL8 to within $\pm 1 \%$ and $\pm 0.4 \%$, respectively, the yields obtained with Magic deviate quite strongly, being too low by more than $20 \%$ at small depth, and too large by more than $50 \%$ at depths $>2 \mathrm{~nm}$. The differences are less pronounced for the total sputtering yield determined by summing up the differential yields in all depth intervals up to $5 \mathrm{~nm}(\mathrm{Y}=2.25,2.42,2.45$ and 2.46 for Magic, GM8, GM16 and GL8, respectively). The calculation times relative to GL8 were 43\% (Magic), 91\% (GM8) and 105\% (GM16). Based on these findings we decided to perform all calculations with GL8.

\section{Results and discussion}

\subsection{Dependence of differential sputtering yields on the position of the primary knock-on atom}

Given the situation sketched in Fig. 1, one might wonder to what extent the position $x_{0}$ of the first struck atom $\mathrm{P}$ will affect differential and total sputtering yields as well as the mean depth of origin. To address the issue, SDTrimSP simulations were performed for Xe impact on a light (Si) as well as a heavy-atom (W) target using a comparatively low impact energy of $1 \mathrm{keV}$ so that any effect near the surface should become evident. Three different possibilities were considered, $\mathrm{P}$ at (i) $x_{0}=0$, (ii) $x_{0}=\lambda_{0}$, or (iii) $x_{0}$ randomly varied between 0 and $\lambda_{0}$ (the case chosen in the work of Shulga and Eckstein [5]). The results are presented in Fig. 3. Considering data for depths $>\lambda_{0}$ first, panel (a) shows that the differential yields are identical within computational uncertainty. The absence of a noticeable difference may be attributed to the fact that the energy loss experienced by the projectile on traversing a layer of thickness $\lambda_{0}$ is too small to affect the evolution of the collision cascade significantly. There is, however, a sizable effect at depths $<\lambda_{0}$, as the data in Fig. 3 (b) show ( $\lambda_{0}=0.272$ and $0.251 \mathrm{~nm}$ for Si and $\mathrm{W}$, respectively). Notably the data for case $x_{0}=\lambda_{0}$ 
deviate quite significantly from the other two cases. This could mean that the collisions leading to the emission of atoms from the depth interval $0 \leq x \leq \lambda_{0}$, were mostly confined to a narrow region close to the surface. The difference between the other two cases is small, less than $2 \%$ in terms of sputtering yield and less than $5 \%$ for the mean depth of origin. Hence, in order to allow a comparison with the results of [5], we chose to perform all other calculations with atom $\mathrm{P}$ at randomly chosen positions in the range $0 \leq x_{0} \leq \lambda_{0}$.

\subsection{Dependence of differential sputtering yields on surface binding energy}

To explore the dependence of differential sputtering yields $\psi(x)$ on the surface binding energy $E_{\mathrm{s}}$, calculations were performed for $10 \mathrm{keV} \mathrm{Xe} \mathrm{impact} \mathrm{on} \mathrm{Si}$, Cs, and W, with $E_{\mathrm{s}}$ varied deliberately between 0.20 and $8.8 \mathrm{eV}$. A representative set of results for $\mathrm{Si}$ is compiled in Fig. 4. In the main panel (a) the data are presented on a logarithmic scale, the inserted panel (b) shows examples of reduced differential yields, $\psi_{\mathrm{n}}(x)=Y^{-1} \mathrm{~d} Y / \mathrm{d} x$, on a linear scale. Two aspects not addressed in prior studies [5,25] deserve attention. First, $\psi(x)$ exhibits maxima at depth between 0 and about $0.1 \mathrm{~nm}$, with heights $(\mathrm{d} Y / \mathrm{d} x)_{\mathrm{pk}}$ increasing almost inversely with $E_{\mathrm{s}}$, notably for $E_{\mathrm{s}}<2 \mathrm{eV}$ (for details see Fig. 5). In accordance with previous work [5], a large fraction of sputtered atoms may originate from depths less than $\lambda_{0} / 2$. As Fig. 4(b) shows, this fraction increases with decreasing $E_{\mathrm{s}}$. Second, at depths exceeding about $0.5 \mathrm{~nm}$, the effect of $E_{\mathrm{s}}$ on $\psi(x)$ vanishes rapidly, to become almost undetectable for $x>2 \mathrm{~nm}$. The Si recoil atoms originating in this depth range must have received a significant amount of energy in the collision cascade $(>100 \mathrm{eV})$, together with the 'right' momentum in the direction of the surface, so that they were not only able to propagate to the surface but also to overcome the surface potential barrier without any problems. As a result, $\psi(x)$ was essentially independent of $E_{\mathrm{s}}$.

The peak differential sputtering yield and the integral (total) yield exhibit a similar, but not exactly the same dependence on $E_{\mathrm{s}}$, see Fig. 5. The two sets of data calculated with $E_{\text {cut }}=0.2 \mathrm{eV}$ tend to become directly proportional to $1 / E_{\mathrm{s}}$ (as indicated by dash-dotted lines), but at opposite ends of the range of $E_{\mathrm{s}}$-values studied, low end for $(\mathrm{d} Y / \mathrm{d} x)_{\mathrm{pk}}$, high end for $Y$. These finer details appear to be associated with the changes in the shape and the position of the peak exemplified in Fig. 4(b). Also shown in Fig. 5 are data calculated for $E_{\text {cut }}$ $=1.5 \mathrm{eV}$. For $E_{\mathrm{s}}>1.5 \mathrm{eV}$, the results are the same as for $E_{\text {cut }}=0.2 \mathrm{eV}$. As $E_{\mathrm{s}}$ is reduced below $1.5 \mathrm{eV}$, however, both $(\mathrm{d} Y / \mathrm{d} x)_{\mathrm{pk}}$ and $Y$ start to fall below the expected behaviour, to arrive at a constant level at $E_{\mathrm{s}} \approx$ $E_{\text {cut }} / 2$. This source of error does not seem to have been fully appreciated in prior static or dynamic TRIM 
calculations in which the effects of $E_{\mathrm{s}}$ were studied. In order to avoid uncertainties, we strongly suggest that the value of $E_{\text {cut }}$ used in such calculations should always be quoted ( $E_{\text {cut }}$ should always be less than $E_{\mathrm{s}}$ ).

\subsection{Dependence of the mean depth of origin on surface binding energy}

Using Eq. (2), the mean depth of origin, $\langle x\rangle$, for $10 \mathrm{keV}$ Xe impact on $\mathrm{Si}, \mathrm{Cs}$ and $\mathrm{W}$ was calculated from differential yields simulated for a wide range of $E_{\mathrm{s}}$-values. The results obtained for the respective (natural) number densities $N=N_{\text {nat }}$ are presented in Fig. 6. The data exhibit a common trend, i.e., $\langle x\rangle$ increases monotonically with increasing $E_{\mathrm{s}}$. However, for the same $E_{\mathrm{s}},\langle x\rangle_{\mathrm{Cs}}$ is much larger than $\langle x\rangle_{\mathrm{W}}$ and $\langle x\rangle_{\mathrm{Si}}$. Given the fact that $N_{\mathrm{Cs}}\left(0.85 \times 10^{22} \mathrm{~cm}^{-3}\right)$ is much smaller than $N_{\mathrm{W}}\left(6.31 \times 10^{22} \mathrm{~cm}^{-3}\right)$ and $N_{\mathrm{Si}}\left(4.99 \times 10^{22}\right.$ $\mathrm{cm}^{-3}$ ), the suspicion was that one should discuss $\langle x\rangle$ not in units of distance but rather in terms of the areal density $\langle x\rangle N$, i.e., the number of atoms per unit area. This assumption is strongly supported by the results of Fig. 6(b) which show that, when presented as the product $\langle x\rangle N$, the DoO depends on $E_{\mathrm{s}}$ in an almost universal manner. The results can be fitted reasonably well by a relation of the form $\langle x\rangle N \propto E_{s}^{p}$, with $p=$ $0.26 \pm 0.03$. To our knowledge, this kind of $E_{\mathrm{s}}$-dependence has not been reported before. It may be worth noting, however, that Shulga [26] tried arrive at a universal description of density and binding effects in lowenergy sputtering yields $(\leq 1 \mathrm{keV})$ by way of replacing $E_{\mathrm{s}}$ in the denominator of Eq. (4) by $E_{s}^{q}$. The resulting $q$-values ranged between 0.75 and 1.35 .

An important aspect is that the power $p$ was found to depend on the impact energy. Results for the $E_{\mathrm{s}^{-}}$ dependence of $\langle x\rangle$ for Xe bombarded $\mathrm{Ag}\left(N=N_{\mathrm{Ag}}\right)$ are summarized in Fig. 7(a). The $p$-values quantifying the simplified power-law dependence range from 0.1 at a $\mathrm{Xe}$ energy of $0.1 \mathrm{keV}$ to 0.23 at $10 \mathrm{keV}$. This previously unknown energy dependence aggravated data evaluation in the study of Shulga and Eckstein [5]. They explored the $Z_{2}$-dependence of $\langle x\rangle N$ for $1 \mathrm{keV}$ Ar normally incident on a wide variety of elemental targets, but for tabulated values of the surface binding energy $E_{\mathrm{s}}$. Inspecting their Fig. 11, we have extracted data in those ranges of $Z_{2}$ where $E_{\mathrm{s}}$ varies rapidly. This is commonly the case for alkali metals and their neighbours of higher $Z_{2}$. Results thus derived are compiled in Fig. 7(b). The data are also in accordance with an $E_{\mathrm{s}}^{p}$-dependence with $p=0.14 \pm 0.02$, essentially the same as in Fig. 7(a) for $1 \mathrm{keV} \mathrm{Xe.} \mathrm{Hence} \mathrm{the} \mathrm{power} p$ does not seem to depend much on the mass of the projectile. 


\subsection{Dependence of the mean depth of origin on the number density of the target}

According to the results of Fig. 5(b), $\langle x\rangle N$ constitutes an almost universal parameter for quantifying the DoO of sputtered atoms. However, a small density effect appeared to exist. To address this issue, a set of calculations was performed for different materials, but intentionally assigning a fixed value of $E_{\mathrm{s}}(4.72 \mathrm{eV})$ to the targets. The results are presented in Fig. 8 as solid symbols. Evidently, the $N$-dependence of $\langle x\rangle N$ is in fact very small, notably at intermediate densities between 2 and $8 \times 10^{22} \mathrm{~cm}^{-3}$. The small gradients seen in Fig. 8 depend little, if any, on $E_{\mathrm{s}}$, as illustrated by the two examples for other $E_{\mathrm{s}}$, denoted by open symbols. The observed trends agree well with the data shown as crosses which represent results for $2 \mathrm{keV} \mathrm{Ne}$ on $\mathrm{Si}$ (adapted from Fig. 2 of [5]).

\subsection{Dependence of differential sputtering yields on the impact energy}

As already discussed by Shulga and Eckstein [5,25], the distributions of the depth of origin of sputtered atoms, $\psi(x)$, depend significantly on the impact energy. Examples of results obtained in this study are presented in Fig. 9 for two widely different energies, 0.1 and $10 \mathrm{keV}$. In order to avoid any effect due to the dependence of $E_{\mathrm{s}}$ and $N$, these two parameters were deliberately kept fixed (the same as for $\mathrm{Si}$ ). The differences in impact energy show up mostly in the tails of $\psi(x)$. Whereas the differential yields for $0.1 \mathrm{keV}$ Xe decrease very rapidly at depths $x>\lambda_{0}$, the data for $10 \mathrm{keV} \mathrm{Xe} \mathrm{exhibit} \mathrm{long,} \mathrm{almost} \mathrm{exponential} \mathrm{tails.} \mathrm{One}$ might conclude that at sufficiently high energies, $\psi(x)$ could be approximated by an exponential function, but, as calculations extended to energies as large as $100 \mathrm{keV}$ have shown [25], the distributions cannot be described by universal analytical functions.

At this point it may be helpful to clarify the difference between $\lambda_{0}$ and $N$-scaling. Differential yields were calculated for $\mathrm{W}$ at three different target densities. The results are shown in Fig. 10(a) as a function of the reduced depth $x / \lambda_{0}=x N^{1 / 3}$. The data are reproduced in Fig. 10(b) on a logarithmic scale, but as a function of the depth normalized as $x N$. The difference is obvious. In the form of Fig. (a), the peak of $\psi(x)$ observed at small depth is due to atoms contained within $\lambda_{0}$, irrespective of $N$. This resembles the suggestion of Kelly and 
Oliva [16] who proposed $\langle x\rangle=(0.8 \pm 0.1) \lambda_{0}$. However, according to the results obtained in this study as well as by TRIM.SP [5,25], the cited kind of relation accounts only for atoms sputtered from the first layer, represented by $\lambda_{0}$. Atoms ejected from deeper layers are not included. Their contribution to $\langle x\rangle$ becomes very clear if one uses the depth scaling of Fig. 10(b), i.e., if $\psi(x)$ is presented as a function of the areal density of target atoms. At moderate to large depths in units of $x N$, the tails of $\psi(x)$ turn out to be independent of $N$. This is the reason for the small density effect seen in Fig. 8.

\subsection{Energy and density dependence of low-energy ranges}

The results of Fig. 9 strongly suggest that the tails of the differential sputtering yields and, concurrently, the mean DoO are correlated with the range of recoil atoms set in motion in the direction of the surface. Hence SDTrimSP was also used for extended range calculations at low energies. Of interest here are only those projectile-target combinations with identical atomic number and mass, i.e., $Z_{2}=Z_{1}$ and $M_{2}=M_{1}$. Apart from the absolute value of the ranges, interest was devoted to the density effect. Examples of range distributions are shown in Fig. 11(a). The data show that the areal density is (again) the appropriate measure of ranges in media of different density: Even though, in the two examples considered, the density differed by a factor of three, the range distribution differ in terms of peak position, width and height by only about $20 \%$.

Scaled mean projected ranges, $\left\langle x_{\mathrm{p}}\right\rangle N$, derived from the calculated range distributions, are presented in Fig. 11(b) as a function of the target density, for 40 and $100 \mathrm{eV} \mathrm{Si} \mathrm{in} \mathrm{Si} \mathrm{and} \mathrm{W} \mathrm{in} \mathrm{W.} \mathrm{Evidently,}$ $\left\langle x_{\mathrm{p}}\right\rangle N$ decreases comparatively little with increasing target density. It is interesting to note that the gradient is almost the same for the two projectile energies as well as for the two targets of different atomic number. On a relative scale, the changes of $\left\langle x_{\mathrm{p}}\right\rangle N$ with increasing density are smaller the higher the energy. These observations may reflect the fact that, as the density decreases, collisions between atoms become possible which involve only the tail region of the interaction potential.

\subsection{Correlation between mean depth of origin and mean range}

At a fixed energy, the results of Figs. 9 showed that differential yields depend very little on the target atomic number, provided $Z>30$. A detailed comparison of the $Z$-dependence of $\langle x\rangle$ and $\left\langle x_{\mathrm{p}}\right\rangle$ is presented 
in Fig. 12. The similarity of the two sets of data is remarkable, providing convincing evidence that the mean depth of origin of sputtered atoms is very closely related to the range of recoils mobilized in the direction of the bombarded surface. In accordance with the arguments outlined in the preceding paragraph, any attempt to assign some mean energy to the recoils is not meaningful because the mean depth of origin constitutes an average over the trajectory of atoms starting at a wide variety of depths, with widely different energies. Based on a direct comparison of $\langle x\rangle$ and $\left\langle x_{\mathrm{p}}\right\rangle$, the data in Fig. 12 could be interpreted very grossly as saying that the atoms sputtered by bombardment with $10 \mathrm{keV}$ Xe from sizable depth started with a mean energy between 20 and $25 \mathrm{eV}$.

A comparison with the results reported by Shulga and Eckstein is again of interest, here the $Z_{2}$ dependence of $\langle x\rangle N$. With $p$ known, we have scaled the published data [5] to a common value of $E_{\mathrm{s}}$, i.e., the original values of $\langle x\rangle N$ were multiplied by $\left(E_{\mathrm{s}, \text { ref }} / E_{\mathrm{s}}\right)^{0.14}$, with $E_{\mathrm{s}, \text { ref }}$ somewhat arbitrarily set to $6.4 \mathrm{eV}$. The results of this exercise are presented in Fig. 13 as solid circles, together with the original data (open triangles). Having removed the $E_{\mathrm{s}}$-dependence of $\langle x\rangle N$, the data of Fig. 13 exhibit the same smooth $Z_{2^{-}}$ dependence as the present results in Fig. 13. The 'oscillation' in $\langle x\rangle N$ reported in the previous study [5] are largely, if not fully, due to the $E_{\mathrm{s}}$-dependence.

\subsection{Preferred method of data presentation}

As already discussed with reference to Fig. 1, calculations of depth differential yields with SDTrimSP involve the problem that the topmost layer is spread out over a depth equivalent to $\lambda_{0}$. This has the consequence that any number derived for $\langle x\rangle$ or $\langle x\rangle N$ is difficult to interpret and to compare with experimental data. Example: What does it mean if the calculations yield $\langle x\rangle=\lambda_{0}$ ? Is this number meant to say that, on average; all sputtered atoms originate in the topmost layer or from the first two layers? The difficulty in answering the question becomes even more evident if we consider the case $\langle x\rangle=0.5 \lambda_{0}$. This would be the result if $\psi(x)$ had a box-type form, extending in depth from 0 to $\lambda_{0}$. Following Shapiro et al. [15], one could argue that this is equivalent to emission of all atoms from the first layer. However, if one would extrapolate the published energy dependence of $\langle x\rangle[5]$ to below $100 \mathrm{eV},\langle x\rangle$ turns out to be less than 
$0.5 \lambda_{0}$. Should this be interpreted as emission from a depth equivalent to less than the topmost layer? Such a result would clearly be unphysical.

To get out of this dilemma we go back to the method of data presentation used previously to quantify experimental data $[2,3]$. The authors quoted the fraction of sputtered atoms originating from the topmost layer, the remaining fraction being attributed to emission from deeper layers. Adopting this approach, we have evaluated the calculated differential yields to determine the fraction of sputtered atoms originating from the depth interval $0 \leq x \leq \lambda_{0}$. The results are presented in Fig. 14. To illustrate the close correlation with the $\langle x\rangle$-data in Fig. 12, the left-hand scale quantifies the fraction emitted from depths $\mathrm{x} \geq \lambda_{0}$. For the sake of completeness, the right-hand vertical scale indicates the fraction emitted from $0 \leq x \leq \lambda_{0}$, the numbers decreasing from bottom to top. As expected, the fraction emitted from below the topmost layer is smallest at very low bombardment energies, with a minimum of about $2 \%$ for $40 \mathrm{eV} \mathrm{Cs}$ on $\mathrm{Cs}\left(Z_{2}=55\right)$. For $10 \mathrm{keV} \mathrm{Xe}$, on the other hand, about $75 \%$ is predicted to be emitted from the topmost layer for targets of medium and high atomic number. This result compares favourably with experimental data. For sputtering of $\mathrm{Cu}$ from $\mathrm{Ru}(0001)$ by normally incident $5 \mathrm{keV}$ Ar, Burnett et al. [2] reported that $66 \pm 12 \%$ of the sputtered atoms originate from the 'first layer'. From an analysis of sputtering a liquid Ga-In eutectic alloy by Ar at normal incidence, Dumke et al. [3] concluded that $85 \%$ of the sputtered atoms originated in the 'surface monolayer' at $15 \mathrm{keV}, 70 \%$ at $25 \mathrm{keV}$. Given the uncertainties associated with insufficient knowledge of the surface binding energies, the agreement between experimental and calculated data may be considered reasonable.

\section{Conclusion}

In this study we have clarified several open questions that evolved from previous TRIM.SP simulations of the distributions of the depth of origin of sputtered atoms, $\psi(x)$. It was shown that the dependence of $\psi(x)$ on target properties can only be unravelled if the effect of the surface binding energy is evaluated independently. If the surface binding energy is kept fixed, the large-depth part of $\psi(x)$ and the mean depth of origin, $\langle x\rangle$, attain an almost universal form when converted to areal densities, $\psi(x N)$ and $\langle x N\rangle$. Previous attempts to identify a correlation between $\langle x\rangle$ and the target density $N$ were not addressing the issue properly. Based on the observation that $\langle x\rangle$ and $\left\langle x_{\mathrm{p}}\right\rangle$ exhibit largely the same dependence on the target atomic number, we argue that the distribution of the depth of origin is determined by the range of recoil 
atoms set in motion in the direction of the surface. Problems associated with the distribution of surface atoms within the concept of SDTrimSP and TRIM.SP lead to the conclusion that calculated data should preferably be presented by quoting the fraction of atoms originating within the depth equivalent to the mean atomic distance (or the mean free path). Results obtained at a moderate bombardment energy of $10 \mathrm{keV}$ fall into the range of data derived from experimental studies. 
Fig. 1. Schematic illustration of a series of collisions in SDTrimSP simulations, terminating in the ejection of atom F. The collisions are projected into the plane of drawing. For details see text.

Fig. 2. Comparison of differential sputtering yields of silicon, calculated by use of three different integration methods for evaluating the scattering integrals in binary collisions. The ratios of the data are also shown.

Fig. 3. Dependence of differential sputtering yields on the assumed position of the first struck atom, for two different target materials. (a) Logarithmic, (b) linear yield scale.

Fig. 4. (a) Differential sputtering yields of silicon for a wide range of assumed surface binding energies. (b) Comparison of normalized yields at shallow depth.

Fig. 5. Peak differential and total sputtering yields versus the surface binding energy, for two values of the cut-off energy. The dash-dotted lines represent yield changes proportional to the inverse surface binding energy.

Fig. 6. (a) Mean depth of origin $\langle x\rangle$ versus the assumed surface binding energy, for three different target of natural number density. The same data as in (a), but as the product $\langle x\rangle N$.

Fig. 7. Scaled mean depth of origin $\langle x\rangle N$ versus the surface binding energy. (a) Ag bombarded with Xe at three different energies, (b) selected targets bombarded with $1 \mathrm{keV}$ Ar. The data in (b) were extracted from Fig. 11 in ref. 5.

Fig. 8. Scaled mean depth of origin $\langle x\rangle N$ versus the assumed number density of three different targets, for $10 \mathrm{keV}$ Xe bomardment. The solid and the open data points relate to different surface binding energies. Data from ref. 5 for $2 \mathrm{keV} \mathrm{Ne}$ on $\mathrm{Si}$ are shown for comparison. 
Fig. 9. Normalized differential sputtering yields for different projectile-target combinations at two widely different impact energies.

Fig. 10. Normalized differential sputtering yields of tungsten for three different assumed target densities. Data plotted as a function of (a) the reduced depth $x / \lambda_{0}$ and (b) the scaled depth $x N$.

Fig. 11. (a) Range distributions of $40 \mathrm{eV} \mathrm{Si} \mathrm{in} \mathrm{Si} \mathrm{for} \mathrm{two} \mathrm{different} \mathrm{assumed} \mathrm{target} \mathrm{densities.} \mathrm{(b)} \mathrm{Scaled} \mathrm{mean}$ projected ranges versus the assumed density of Si and $\mathrm{W}$.

Fig. 12. Dependence of the mean depth of origin and mean projected ranges on the atomic number of the target. All targets were assumed to feature the same density.

Fig. 13. Dependence of the scaled mean depth of origin on the atomic number of Ar bombarded targets. Open triangles: Data extracted from Fig. 11 in ref. 5; solid circles: after correction for the effect of the surface binding energy.

Fig. 14. Fraction of atoms sputtered from the depth interval nominally equivalent to the topmost layer (righthand scale) or from depths beyond the topmost layer (left-hand scale), versus the target atomic number. All targets were assigned the same density and surface binding energy. 
[1] S. Prigge, E. Bauer, Adv. Mass Spectrom. 8 (1980) 543.

[2] J.W. Burnett, J.P. Biersack, D.M. Gruen, B. Jørgensen, A.R. Krauss, M.J. Pellin, E.L. Schweitzer, J.T. Yates, Jr., C.E. Young, J. Vac. Sci. Technol. A 6 (1988) 2064.

[3] M.F. Dumke, T.A. Tombrello, R.A. Weller, R.M. Housley, E.H. Cirlin, Surf. Sci. 124 (1984) 407.

[4] K. Wittmaack, Phys. Rev. B 56 (1997) R5701.

[5] V.I. Shulga, W. Eckstein, Nucl. Instr. and Meth. B 145 (1998) 492.

[6] M.T. Robinson, J. Appl. Phys. 54 (1983) 2650.

[7] V.I. Shulga, Radiat. Eff. 70 (1983) 65.

[8] P. Sigmund, Phys. Rev.184 (1969) 383.

[9] M. Vicanek, J.J. Jimenez Rodriguez, P. Sigmund, Nucl. Instr. and Meth. B 36 (1989) 124.

[10] K. Wittmaack, Phys. Rev. B 68 (2003) 235211.

[11] J. Bohdansky, Nucl. Instr. and Meth. B 2 (1984) 587.

[12] H.H. Andersen, H.L. Bay, J. Appl. Phys. 46 (1975) 2416.

[13] J. Bohdansky, J. Roth, H.L. Bay, J. Appl. Phys. 51 (1980) 2861.

[14] G. Falcone, P. Sigmund, Appl. Phys. 25 (1981) 307.

[15] M.H. Shapiro, E. Trovato, T.A. Tombrello, Nucl. Instr. and Meth. B 180 (2001) 58.

[16] R. Kelly, A. Oliva, Nucl. Instr. and Meth. B 13 (1989) 283.

[17] A. Mutzke, R. Schneider, W. Eckstein, R. Dohmen, Max-Planck-Institut für Plasmaphysik, IPP-Report 12/8 (2011).

[18] J.P. Biersack, W. Eckstein, Appl. Phys. A 34 (1984) 73.

[19] W. Möller, W. Eckstein, Nucl. Instr. and Meth. B 2 (1984) 814.

[20] W.D. Wilson, L.G. Haggmark, J.P. Biersack, Phys. Rev. B 15 (1977) 2458.

[21] W. Eckstein, Computer Simulation of Ion-Solid Interactions, Springer Series in Material Science, Vol. 10, Springer, Berlin, 1991.

[22] J.P. Biersack, L.G. Haggmark, Nucl. Instr. and Meth. 174 (1980) 257.

[23] K. Mehler, J. Reine Angew. Math. 63 (1864) 152.

[24] Z. Kopal, Numerical Analysis, Chapman and Hall, London, 1961.

[25] W. Eckstein, V.I. Shulga, Nucl. Instr. and Meth. B 164-165 (2000) 748.

[26] V.I. Shulga, Nucl. Instr. and Meth. B 179 (2001) 485. 


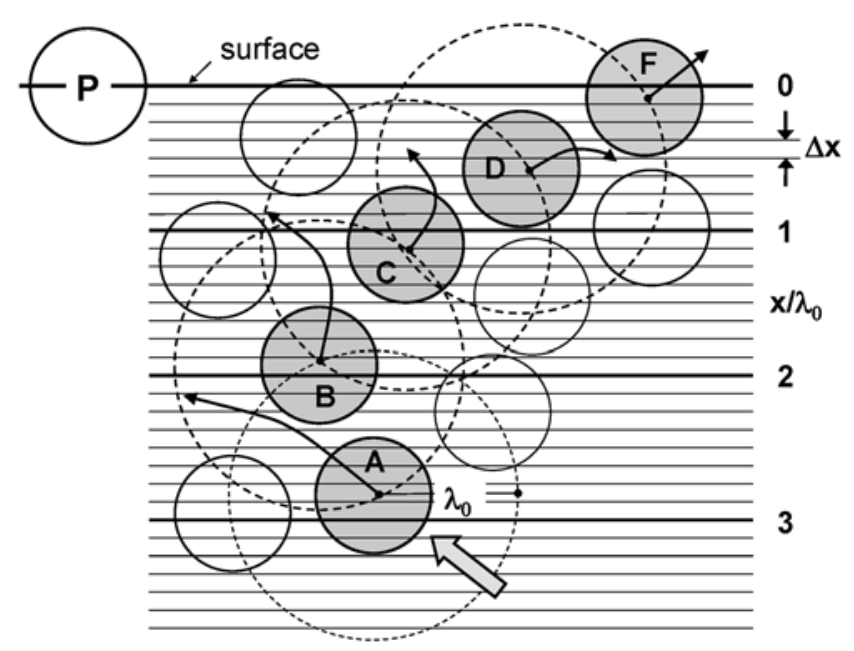

Fig. 1

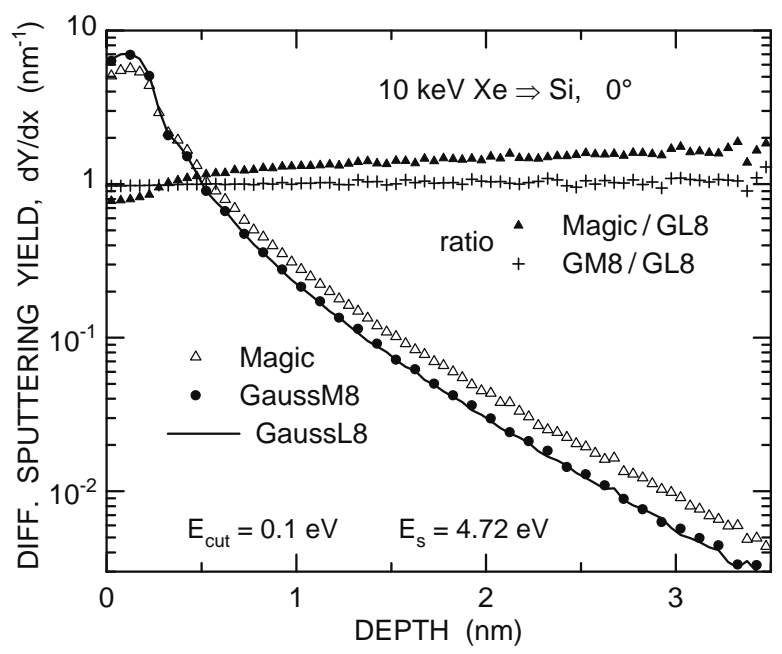

Fig. 2
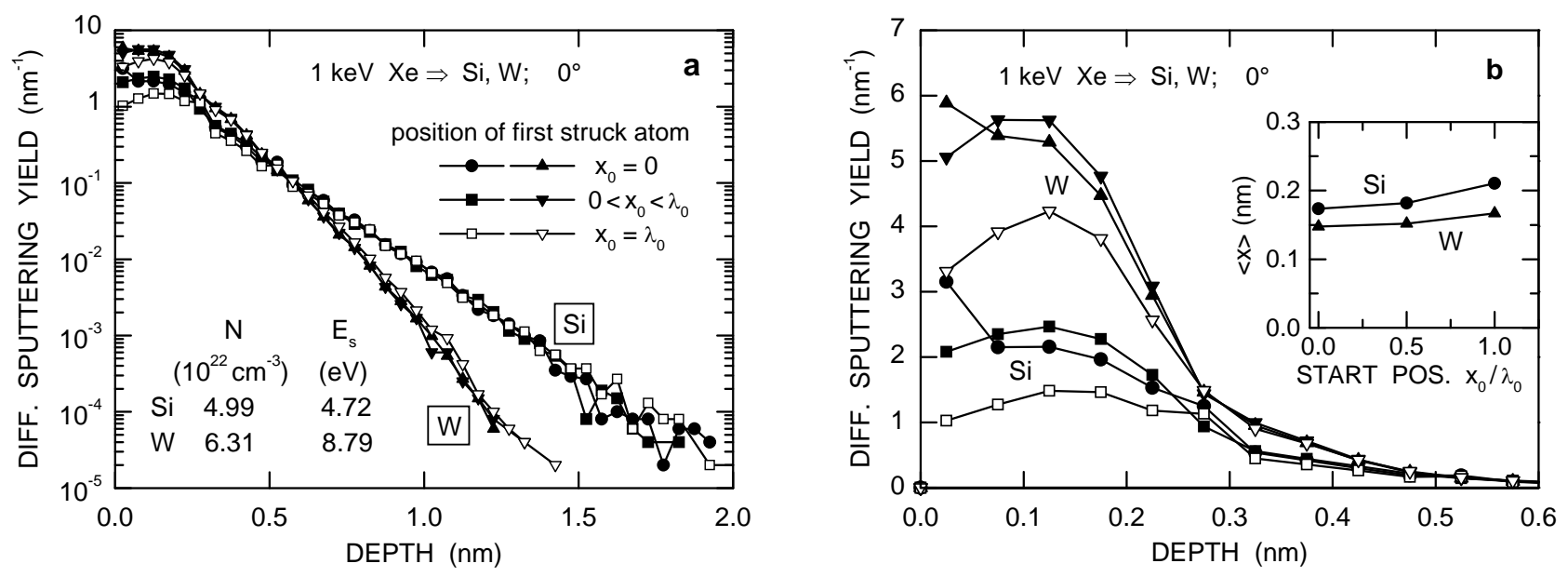

Fig. 3 


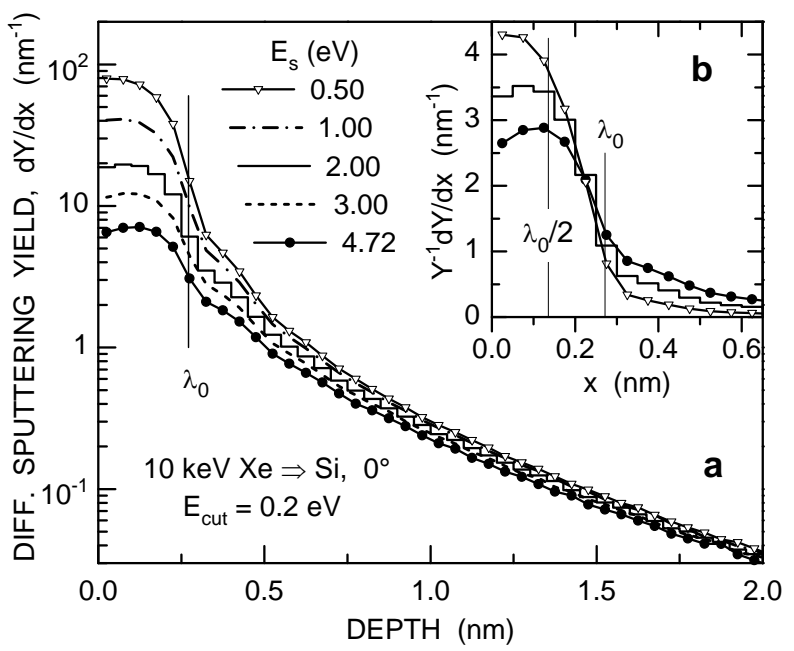

Fig. 4

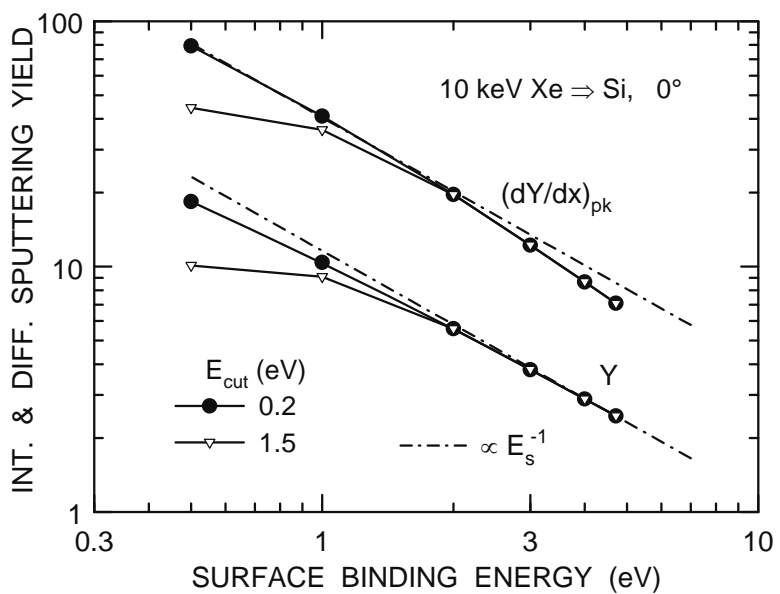

Fig. 5

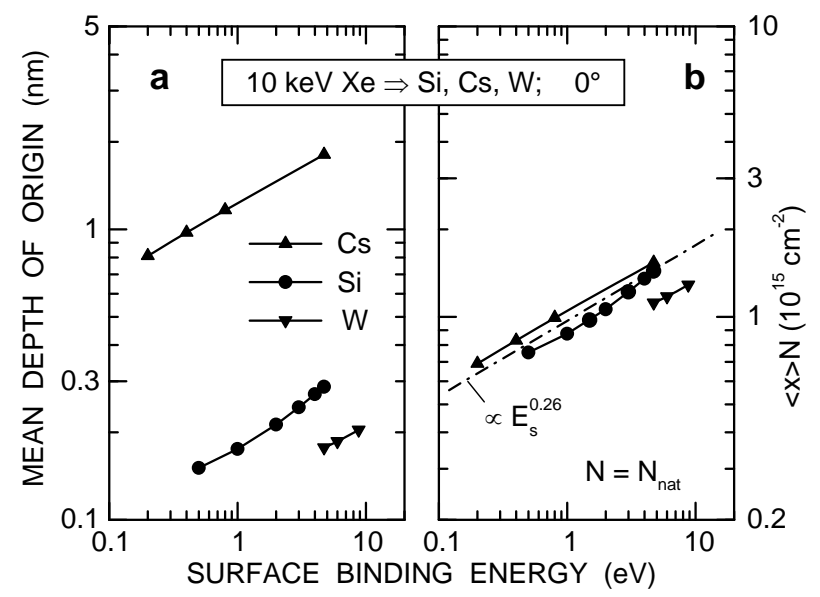

Fig. 6 

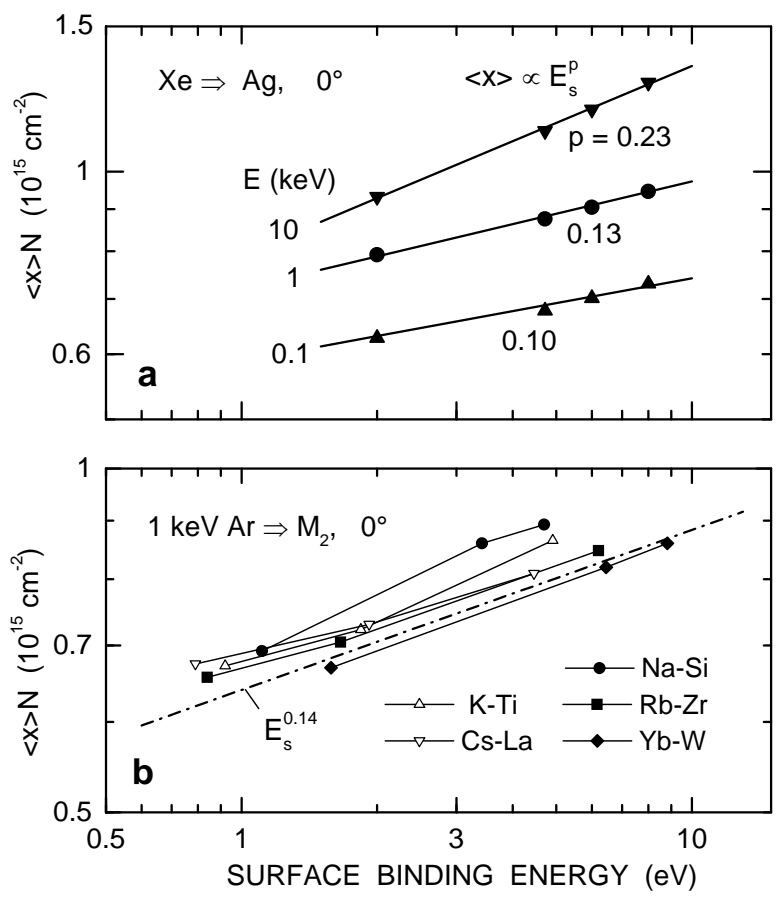

Fig. 7

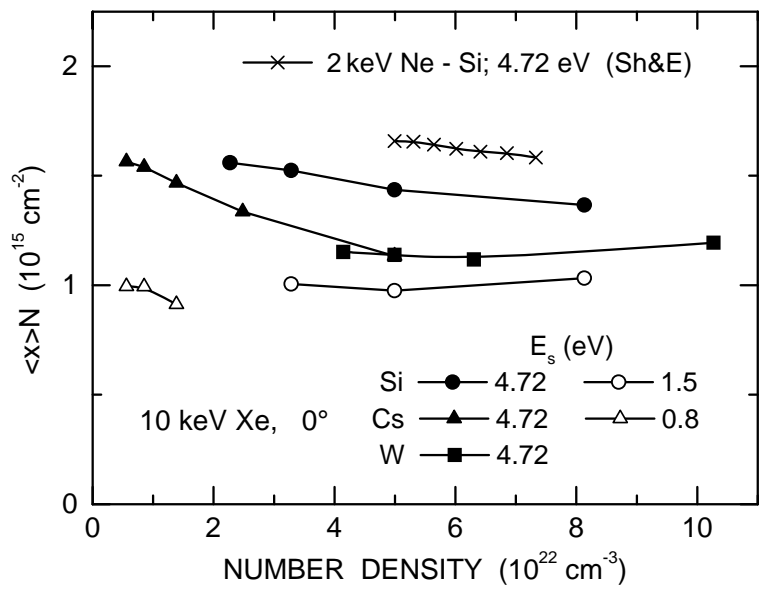

Fig. 8



Fig. 9 

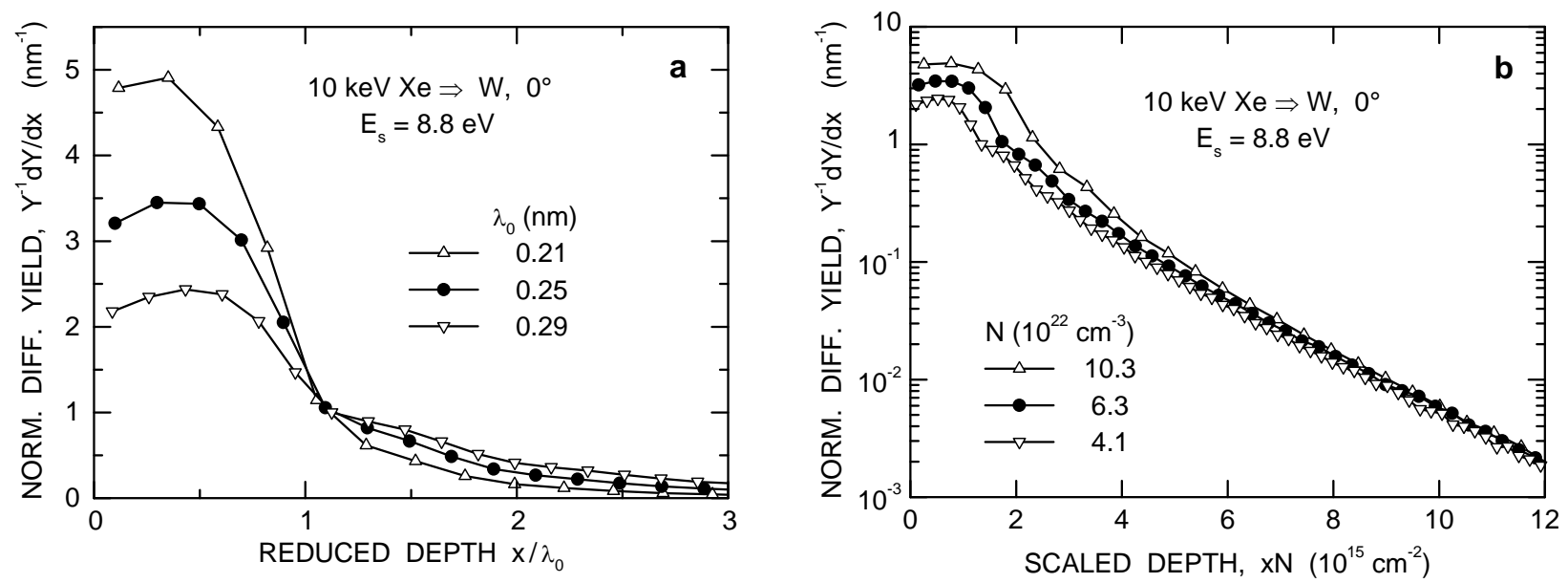

Fig. 10

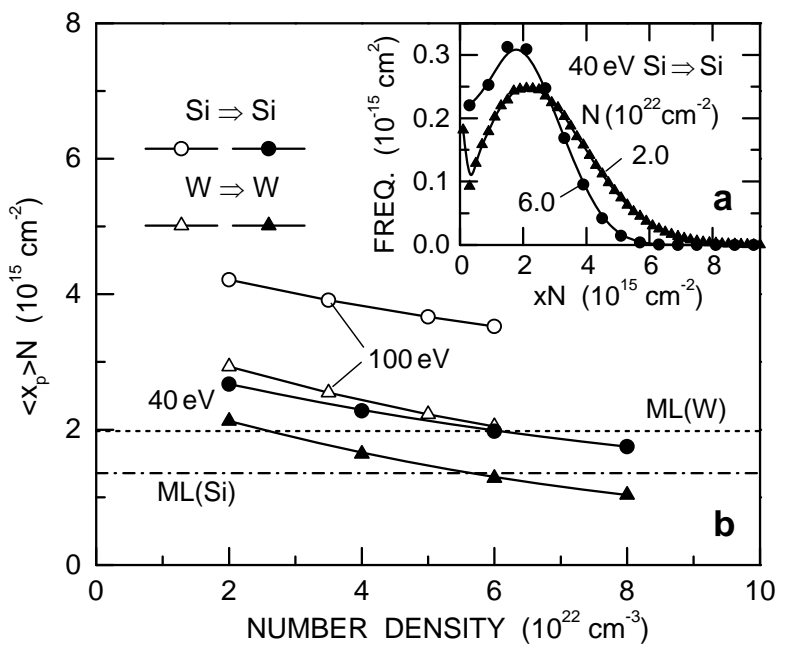

Fig. 11

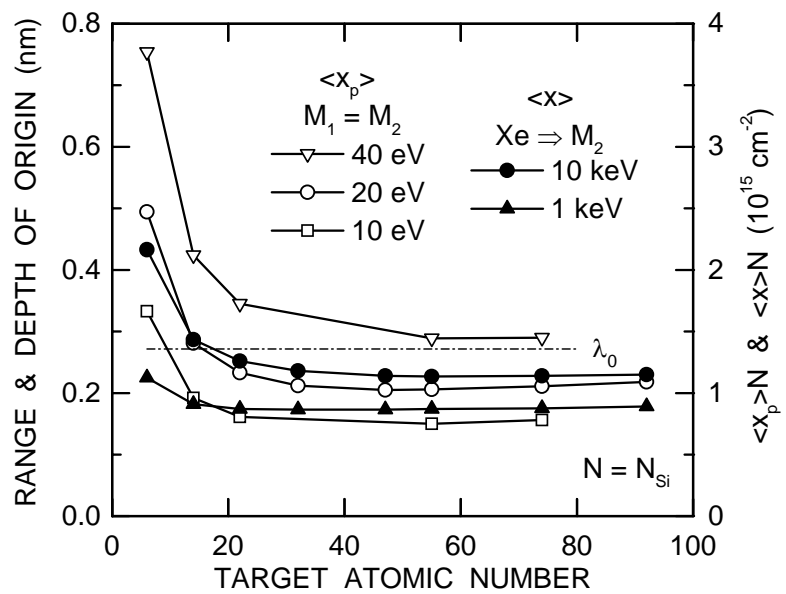

Fig. 12 




Fig. 13

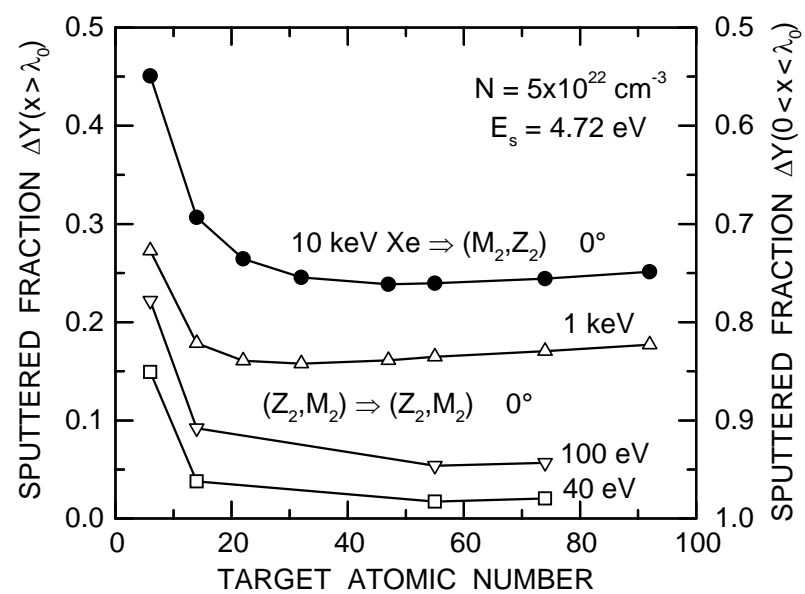

Fig. 14 